\title{
High vaccination efficiency of low-affinity epitopes in antitumor immunotherapy
}

\author{
David-Alexandre Gross, ${ }^{1}$ Stéphanie Graff-Dubois, ${ }^{1}$ Paule Opolon, ${ }^{2}$ Sébastien Cornet, ${ }^{1}$ \\ Pedro Alves, ${ }^{1}$ Annelise Bennaceur-Griscelli, ${ }^{3}$ Olivier Faure, ${ }^{1}$ Philippe Guillaume, ${ }^{4}$ \\ Hüseyin Firat, ${ }^{5}$ Salem Chouaib, ${ }^{1}$ François A. Lemonnier, ${ }^{5}$ Jean Davoust, ${ }^{6}$ \\ Isabelle Miconnet, ${ }^{1}$ Robert H. Vonderheide, ${ }^{7}$ and Kostas Kosmatopoulos ${ }^{1}$
}

${ }^{1}$ Institut National de la Santé et de la Recherche Médicale Unité 487,
${ }^{2}$ Centre National de la Recherche Scientifique Unité Mixte de Recherche 8121, and
${ }^{3}$ Institut National de la Santé et de la Recherche Médicale Unité 362, Institut Gustave Roussy, Villejuif, France
${ }^{4}$ Ludwig Institute for Cancer Research, Lausanne Branch, University of Lausanne, Epalinges, Switzerland
${ }^{5}$ Unité d'Immunité Cellulaire Antivirale, Institut Pasteur, Paris, France
${ }^{6}$ Centre National de la Recherche Scientifique Unité Mixte de Recherche 8115, Généthon, Evry, France
${ }^{7}$ Abramson Family Cancer Research Institute, University of Pensylvania School of Medicine, Philadelphia, Pennsylvania, USA

Most of the human tumor-associated antigens (TAAs) characterized thus far are derived from nonmutated "self"-proteins. Numerous strategies have been developed to break tolerance to TAAs, combining various forms of antigens with different vectors and adjuvants. However, no study has yet determined how to select epitopes within a given TAA to induce the highest antitumor effector response. We addressed this question by evaluating in HLA-A*0201-transgenic HHD mice the antitumor vaccination efficacy of high- and low-affinity epitopes from the naturally expressed murine telomerase reverse transcriptase (mTERT). Immunity against low-affinity epitopes was induced with heteroclitical variants. We show here that the CTL repertoire against high-affinity epitopes is partially tolerized, while that against low-affinity epitopes is composed of frequent CTLs with high avidity. The highaffinity p797 and p545 mTERT epitopes are not able to protect mice from a lethal challenge with the mTERT-expressing EL4-HHD tumor. In contrast, mice developing CTL responses against the p572 and p988 low-affinity epitopes exhibit potent antitumor immunity and no sign of autoimmune reactivity against TERT-expressing normal tissues. Our results strongly argue for new TAA epitope selection and modification strategies in antitumor immunotherapy applications in humans.

J. Clin. Invest. 113:425-433 (2004). doi:10.1172/JCI200419418.

\section{Introduction}

Since the majority of tumor-associated antigens (TAAs) are nonmutated self-proteins expressed on normal tissues including the thymus $(1,2)$, self-tolerance is a major barrier to the development of $\mathrm{T}$ cell responses capable of tumor rejection. Self-tolerance shapes a TAA repertoire that has been purged of high-avidity CTLs and thus only weakly effective at eliciting in vivo antitumor immunity, as shown for the CTL response

Received for publication July 8, 2003, and accepted in revised form November 25, 2003.

Address correspondence to: David-Alexandre Gross, Généthon, CNRS UMR 8115, 1 bis, rue de l'internationale, 91002 Evry Cedex, France. Phone: 33-1-69-47-25-77; Fax: 33-1-60-77-86-98; E-mail: gross@genethon.fr.

Conflict of interest: The authors have declared that no conflict of interest exists.

Nonstandard abbreviations used: tumor-associated antigen (TAA); telomerase reverse transcriptase (TERT); murine TERT (mTERT); T cell receptor (TCR); relative affinity (RA);

dissociation complex $50\left(\mathrm{DC}_{50}\right)$; incomplete Freund's adjuvant (IFA); chimeric HLA-A*0201 heavy chain (human $\alpha 1, \alpha 2$, and murine $\alpha 3$ ) covalently linked to the human $\beta 2 \mathrm{~m}$ light chain (HHD); phycoerythrin (PE); concanavalin A (ConA); concentration of peptide that gives the half-maximal lysis of target cells $\left(\mathrm{KC}_{50}\right)$. against p53 and tyrosinase epitopes $(3,4)$. Indeed the efficacy of vaccination protocols depends on the avidity of the recruited CTLs $(5,6)$. In the B16 melanoma model, differences in the avidity of CTLs that recognize p15E and TRP2 self-antigens are critically important for their in vitro and in vivo antitumor efficacy (7).

Several different powerful approaches have been pursued to recruit rare high-avidity CTLs and override tolerance. Vaccination has been combined with either enhancers of the immune response such as cytokines (8) and costimulatory molecules (9), or blockade of negative regulators of $\mathrm{T}$ cell response such as CTLA-4 and regulatory $\mathrm{T}$ cells (10). Cellular or gene therapy protocols based on dendritic cells (11), exosomes (12), heat shock proteins (13), or recombinant virus (14) have also been successful in the induction of protective immunity in mice. Various forms of the antigen were also compared, such as peptide, protein, plasmid DNA, or RNA derived from the autologous sequence or a xenogeneic one $(11,15)$. However, no study has yet determined which epitopes within a given TAA are the best target in antitumor vaccination.

Many influences contribute to the immunodominance process: the binding of peptides to class I molecules, the efficiency of antigen presentation including 
competition between determinants and alleles, and differential regulation of proteasome cleavages $(16,17)$. Self-tolerance concerns mainly the $\mathrm{T}$ cell repertoire, restricted by dominant self-epitopes and, rarely, by the cryptic epitopes as already shown $(18,19)$. Since the CTL repertoire specific for cryptic epitopes might escape the negative selection process, high-avidity CTLs against cryptic epitopes should be recruited with ease and produce a potent antitumor immunity. It is difficult, however, to predict the variables that govern epitope crypticity and dominance within a given TAA. Since dominant determinants are often good binders while cryptic ones are poor binders, we decided here to analyze the antitumor vaccination potential of CTLs raised against high- and low-affinity epitopes.

Low-affinity epitopes are poorly immunogenic if immunogenic at all, however. To circumvent this problem, we have created modified heteroclitical peptides to enhance their affinity for class I molecules while preserving the amino acids that interact with the $\mathrm{T}$ cell receptor (TCR) $(20,21)$. Vaccination with such variants of low-affinity epitopes of the influenza nucleoprotein induces a potent anti-influenza PR8 protective immunity in C57BL/6 mice (22). Furthermore, the majority of low-affinity peptides restricted by HLA-A*0201 modified with a tyrosine in the first position (P1Y) have enhanced capacity to bind this MHC molecule and become highly immunogenic in HHD mice (23). This approach allowed us to identify new epitopes of the human Her-2/Neu proto-oncogene and telomerase reverse transcriptase (TERT) antigens (24).

TERT is considered a potential target for cancer immunotherapy, since malignant cells preferentially express this antigen, which is present in more than $80 \%$ of human tumors. Its normal expression is limited to specific normal cells and tissues, including germinalcenter $\mathrm{B}$ cells, activated $\mathrm{B}$ and $\mathrm{T}$ cells, the thymus, the gut, and the bone marrow (25-27). Numerous epitopes have been identified thus far that are restricted either by MHC class I (A2.1, A3, and A24) or by class II molecules (DR7) (28-31). However, in vivo antitumor immunity against TERT has been observed only using TERT RNA-transfected dendritic cells (32), and the TERT rejection epitopes are still unknown. We evaluate here in HLA-A*0201-transgenic HHD mice the antitumor efficacy of high- and low-affinity epitopes of the murine TERT (mTERT). The size and avidity of their repertoire correlate with their antitumor efficacy. In addition, successful tumor vaccination with lowaffinity mTERT epitopes does not induce autoreactive responses against normal tissues expressing mTERT.

\section{Methods}

Animals. The HLA-A*0201-transgenic HHD mice have been previously described (20).

Cells. Murine RMAS/HHD, RMA/HHD, EL4S3Rob/HHD (EL4/HHD), RMA, and EL4S3-Rob cells have been previously described (20). Cells were grown in RPMI 1640 medium supplemented with 10\% FCS.
Peptides. Peptides were synthesized by Synt:em (Nîmes, France).

Measurement of peptide relative affinity (RA) for HLA$A * 0201$. The protocol used to measure RA has been described previously (23). Briefly, T2 cells were incubated with various concentrations of peptides $(0.1-100$ $\mu \mathrm{M})$ for 16 hours and then stained with the mAb BB7.2 to quantify the surface expression of HLA-A*0201. For each peptide concentration, the HLA-A*0201-specific staining was calculated as the percentage of the staining obtained with $100 \mu \mathrm{M}$ of the reference peptide HIVpol $_{589}$ (IVGAETFYV). The RA is determined as the ratio between the concentration of each peptide and the concentration of the reference peptide that induces $20 \%$ of HLA-A* 0201 expression.

Assessment of peptide-HLA-A*0201 complex stability. As previously described (23), T2 cells were incubated overnight with $100 \mu \mathrm{M}$ of each peptide at $37^{\circ} \mathrm{C}$ in serum-free medium. Cells were then incubated with Brefeldin A (Sigma-Aldrich, St. Louis, Missouri, USA) at $10 \mu \mathrm{g} / \mathrm{ml}$ for 1 hour, washed, and incubated at $37^{\circ} \mathrm{C}$ during $0,2,4$, or 6 hours in the presence of Brefeldin A at $0.5 \mu \mathrm{g} / \mathrm{ml}$. Cells were stained with BB7.2 mAb. For each time point, peptide-induced HLA-A*0201 expression was calculated as the mean fluorescence of peptide-preincubated $\mathrm{T} 2$ cells minus the mean fluorescence of T2 cells treated in similar conditions in the absence of peptide. Dissociation complex $50\left(\mathrm{DC}_{50}\right)$ was defined as the time required for the loss of $50 \%$ of the HLA-A*0201-peptide complexes stabilized at $t=0$.

Generation of CTL in HHD mice. HHD mice were injected subcutaneously with $100 \mu \mathrm{g}$ of peptide emulsified in incomplete Freund's adjuvant (IFA) in the presence of $150 \mu \mathrm{g}$ of the I-A $\mathrm{A}^{\mathrm{b}}$-restricted HBVcore ${ }_{128} \mathrm{~T}_{\mathrm{H}}$ epitope. After 11 days, spleen cells $\left(5 \times 10^{7}\right.$ cells in $\left.10 \mathrm{ml}\right)$ were stimulated in vitro with peptide $(10 \mu \mathrm{M})$ in RPMI 1640 plus 10\% FCS. The CTL lines were established by weekly in vitro restimulation with irradiated spleen cells in the presence of peptide $(1 \mu \mathrm{M})$ and $50 \mathrm{U} / \mathrm{ml}$ IL-2 (Proleukin, Chiron Corp., Emeryville, California, USA).

Cytotoxic assay. We incubated ${ }^{51} \mathrm{Cr}$-labeled peptidepulsed $\left(37^{\circ} \mathrm{C}\right.$ for 60 minutes) or unpulsed targets $\left(2.5 \times 10^{3}\right.$ cells $)$ with different concentrations of effector cells in a total volume of $200 \mu \mathrm{l}$ at $37^{\circ} \mathrm{C}$ for 4 hours. After incubation, a 100- $\mu \mathrm{l}$ aliquot of supernatant was collected, and radioactivity was measured in a $\gamma$-counter. The percentage of specific lysis was determined as (experimental release - spontaneous release) / (maximal release - spontaneous release).

Histology and immunobistology staining. For histological studies, subcutaneous immunization was performed with mTERT or control peptide $(100 \mu \mathrm{g})$, the HBVcore $_{128} \mathrm{~T}_{\mathrm{H}}$ peptide $(150 \mu \mathrm{g})$, and IFA, and repeated once after 14 days. At different time points after vaccination, necropsy was performed; $4-\mu \mathrm{m}$-thick paraffin sections were then made from the sampled tissues and stained with H\&E-safranin (Merck KGaA, Darmstadt, Germany). For immunohistology, $5-\mu \mathrm{m}$-thick acetonefixed frozen sections were incubated with rat anti-CD4, 
anti-CD8, or anti-CD19 Ab (Pharmingen, San Diego, California, USA). After washing, a rabbit anti-rat Ig Ab and then APAAP complex specific for rat (DAKO Corp., Trappes, France) were added. Alkaline phosphatase activity was revealed with Red Alkaline Phosphatase Substrate Kit I (Vector Laboratories Inc., Burlingame, California, USA).

Flow cytometry. Cells were incubated with the 2.4G2 $\mathrm{mAb}$ for 15 minutes to block $\mathrm{Fc}$ receptor and then stained with anti-B220-APCs, anti-IgD-FITC, antiIgM-phycoerythrin (anti-IgM-PE) (Pharmingen, Becton Dickinson Biosciences, Le Pont de Claix, France) for 30 minutes. For tetramer staining, cells from peripheral blood were stained with $15 \mu \mathrm{g} / \mathrm{ml}$ of PE-coupled HLA-A*0201-p797 (SVFDFFHFL) or HLA$\mathrm{A}^{*} 0201-\mathrm{p} 572 \mathrm{Y}$ (YLFFYRKSV) tetramer in the presence of anti-Fc receptor $\mathrm{Ab}$ (clone 2.4G2) in $20 \mu \mathrm{l} \mathrm{PBS,} 2 \%$ FCS for 1 hour at room temperature. Cells were washed once in PBS with $2 \%$ FCS and then stained with antiCD44-FITC (clone 1M.178), anti-TCR $\beta$-CyChrome (clone H57), and anti-CD8 $\alpha$-APC (clone 53.6.7) (Pharmingen, Becton Dickinson Biosciences) in $50 \mu \mathrm{l}$ of PBS with $2 \% \mathrm{FCS}$ for 30 minutes at $4{ }^{\circ} \mathrm{C}$ and then immediately analyzed in a FACSCalibur (Pharmingen, Becton Dickinson Biosciences).

Telomerase activity assays. Telomerase activity was measured by conventional TRAP assay after CHAPS protein extraction using the TRAPeze kit (Intergen Co., Oxford, United Kingdom). CHAPS extraction was performed using $10^{3}$ primary HHD cells or tumor cells. Real-time quantitative TRAP PCR was performed using ABI PRISM 7700 Detection System (Perkin-Elmer Applied Biosystems, Foster City, California, USA) and SYBRGreen (Applied Biosystems, Foster City, California, USA) as previously described (33) in a total reaction volume of $25 \mu \mathrm{l}$ containing $0.4 \mu \mathrm{g}$ of CHAPS extract. The standard curve was established using serial dilutions of HL60 cell CHAPS extracts (from 1 to $10^{5}$ cells). ELISA. IgM and IgG concentrations in sera were measured by ELISA and determined by comparing the displacement of the dilution curves in the linear interval between standard IgM and IgG at a concentration of $1-10 \mu \mathrm{g} / \mathrm{ml}$ and the serum samples.

ELISPOT assay. Peptide-specific T cells from vaccinated mice were counted by IFN- $\gamma$ ELISPOT as previously described (21). Anti-mouse IFN- $\gamma$ mAb's $(5 \mu \mathrm{g} / \mathrm{ml})$ (Pharmingen, Becton Dickinson Biosciences) were coated onto 96-well nitrocellulose microplates (Multiscreen; Millipore Corp., Molsheim, France). After red cell lysis, freshly isolated splenocytes $\left(3 \times 10^{6}\right.$ cells/well and serial dilutions) were directly cultured with or without $10 \mu \mathrm{M}$ of native peptide. For each cell suspension, concanavalin $\mathrm{A}(\mathrm{Con} \mathrm{A})$ was added $(5 \mu \mathrm{g} / \mathrm{ml})$ as a positive control. After 18 hours, plates were washed three times with PBS-Tween and PBS, incubated 2 hours with biotinylated anti-mouse IFN- $\gamma$ (Pharmingen, Becton Dickinson Biosciences) and 1.5 hours with alkaline phosphatase-conjugated streptavidin (Roche Molecular Biochemicals, Mannheim, Germany). Spots were developed by adding peroxidase substrates, 5-bromo-4,3-indolyl phosphate, and nitroblue tetrazolium (Promega Corp., Madison, Wisconsin, USA) and counted using the automated image analysis system ELISPOT Reader (Autoimmun Diagnostika $\mathrm{GmbH}$, Strassberg, Germany).

In vivo tumor protection assay. HHD mice were vaccinated with $100 \mu \mathrm{g}$ of peptide emulsified in IFA in the presence of $150 \mu \mathrm{g}$ of the I-A $\mathrm{A}^{\mathrm{b}}$-restricted HBVcore ${ }_{128}$ epitope, once and then again 2 weeks later. One week after the second vaccination, they were challenged subcutaneously with $2 \times 10^{4}$ EL4/HHD cells. Tumor volumes were measured with calipers.

\section{Results}

Identification of high-and low-affinity HLA-A*0201-restricted mTERT epitopes. To identify high-affinity mTERT epitopes, we evaluated five peptides (p530, p534, p545, p797, and p876) that had the highest predictive score for HLA-A*0201 binding, based on a peptide-motif scoring system (Table 1) (34). These peptides exhibited a high affinity $(\mathrm{RA}<2)$ and formed stable peptideHLA-A*0201 complexes ( $\mathrm{DC}_{50}>6$ hours). Upon immunization the strong binders p530 and p534 were nonimmunogenic, while the intermediate binders p545, p797, and p876 triggered specific cytotoxic T cell responses in HHD mice (Figure 1a). For p530 and p534, three to five mice per group in three independent experiments have been immunized; this led us to hypothesize that the lack of response may be due to complete tolerance of their CTL repertoire. Next, we generated CTL lines specific for p545, p797, and p876 (CTL $\mathrm{C}_{545}, \mathrm{CTL}_{797}$, and $\mathrm{CTL}_{876}$, respectively) and tested their capacity to recognize endogenously processed mTERT. CTL 545 and $\mathrm{CTL}_{797}$ killed the TERT ${ }^{+}$HLA$\mathrm{A}^{*} 0201^{+}$EL4/HHD but killed neither the TERT ${ }^{+} \mathrm{HLA}^{-}$ $\mathrm{A}^{*} 0201^{-}$EL4S3-Rob cells nor the HLA-A*0201+ ${ }^{+}$TERT $^{+}$ RMAS/HHD control cells, which are deficient for the transporter associated with antigen processing (TAP) transporter deficient. None of the CTL lines against p876 killed any of these targets (Figure 1b). We further checked the capacity of $\mathrm{CTL}_{797}$ to recognize endogenous mTERT in another tumor cell line using RMA/HHD cells (Figure 1c). As with EL4/HHD cells, we found that $\mathrm{CTL}_{797}$ killed TERT ${ }^{+}$HLA-A $^{*} 0201^{+}$ RMA/HHD but not TERT ${ }^{+}$HLA-A*0201- RMA cells.

Next, to identify the low-affinity binding peptides, which may behave as cryptic epitopes, we relied on our previous characterization of two human TERT epitopes (p572 and p988) exhibiting a very low affinity for HLA$A^{*} 0201$, and also present in the mTERT sequence (24). Here we show that these two peptides are nonimmunogenic in their native form but can trigger immune responses when used as $\mathrm{P} 1 \mathrm{Y}$ heteroclitical variants with high affinity for HLA-A*0201 (23). Here, too, the corresponding $\mathrm{CTL}_{572}$ and $\mathrm{CTL}_{988}$, generated in HLA$A^{*}$ 0201-transgenic mice, recognized naturally expressed endogenous mTERT (Figure 1b). As with the dominant peptides, CTL generated in HHD mice vaccinated with 
Table 1

Affinity for HLA-A*0201 of mTERT-derived peptides

\begin{tabular}{lccc}
\hline Peptides & Sequence & RA $^{\mathrm{A}}$ & $\mathrm{DC}_{50}$ (hours) $^{\mathrm{B}}$ \\
p530 & ILATFLFWL & 0.8 & $>6$ \\
P534 & FLFWLMDTY & 0.2 & $>6$ \\
P545 & QLLRSFFLHFL & 1.8 & $>6$ \\
P797 & SLFDFFHFL & 1.5 & $>6$ \\
P876 & FLSTLVHGV & 1.2 & $>6$ \\
P572 & RLFFYRKSV & 25.3 & $<2$ \\
p572Y1 & YLFFYRKSV & 2.2 & 5 \\
P988 & DLQVNSLQTV & 28.6 & $<2$ \\
P988Y1 & YLQVNSLQTV & 2.1 & $>6$ \\
\hline
\end{tabular}

ARelative affinity (RA): concentration of each peptide/concentration of the reference peptide that induce $20 \%$ of HLA-A*0201 expression obtained by $100 \mu \mathrm{M}$ of the reference peptide. ${ }^{B} D C_{50}$ : half-life of the peptide/HLA-A*0201 complex.

p572Y and p988Y peptides killed the EL4/HHD but killed neither the EL4S3-Rob cells nor the RMAS/HHD cells. The HLA-A*0201-restricted recognition of endogenous mTERT by $\mathrm{CTL}_{572}$ was also confirmed here using RMA/HHD cells as targets (Figure 1c).

Together, these results demonstrate that the high-affinity p 545 and p797 and the low-affinity p572 and p988 are HLA-A*0201-restricted epitopes of the mTERT.

Quality of the CTL repertoire specific for high-and low-affinity mTERT epitopes. To analyze the quality of the antitumor CTL repertoire for each peptide, we monitored CTL avidity and frequency, both of which are crucial for the efficacy of tumor immunotherapy (5-7). Since TERT is highly expressed in the thymus, we expected differences in the quality and quantity of the CTL spe- cific for either the high-affinity TERT (p545 and p797) peptides or the low-affinity (p572 and p988) peptides. The frequency of CTLs was evaluated 11 days after vaccination directly ex vivo by ELISPOT assay against target loaded with native peptides. Indeed, we found a greater number of CTLs recognizing the low-affinity epitopes p 572 and p $988(0.65 \% \pm 0.06 \%$ and $0.75 \% \pm 0.08 \%$ of $\mathrm{CD}^{+}$cells, respectively) than recognizing the highaffinity p 545 and p797 $(0.1 \% \pm 0.01 \%$ and $0.3 \% \pm 0.06 \%)$ (Figure 2a). Second, we estimated the CTL avidity from the concentration of peptide that gives the half-maximal lysis $\left(\mathrm{KC}_{50}\right)$ of RMAS/HHD using peptide primed spleen cells stimulated once in vitro with the cognate TERT peptide. To account for the difference in the affinity of the peptides, the measured $\mathrm{KC}_{50}$ was normalized by the RA of individual peptide to HLA$A^{*} 0201$, allowing comparison of TCR avidity for equivalent numbers of HLA-peptide complexes on target cells. Here too, CTLs specific for the low-affinity p572 and p988 epitopes exhibited a higher avidity $(10.5 \pm 6$ $\mathrm{nM}$ and $7.8 \pm 1.3 \mathrm{nM}$ ) than CTLs specific for the highaffinity p545 and p797 epitopes $(83 \pm 35.8 \mathrm{nM}$ and $108.9 \pm 40.6 \mathrm{nM}$ ) (Figure $2 \mathrm{~b}$ ). It is noteworthy that avidity and frequency of CTLs against the low-affinity epitopes were comparable to those of CTLs recognizing the nonprocessed $\mathrm{p} 876$ peptide as well as the influenza virus M58 epitope.

We found qualitative and quantitative differences between CTL repertoires directed against high- and lowaffinity epitopes of the mTERT; these differences could result from deletion mechanisms operating in the thymus or anergy induction due to TAA recognition on
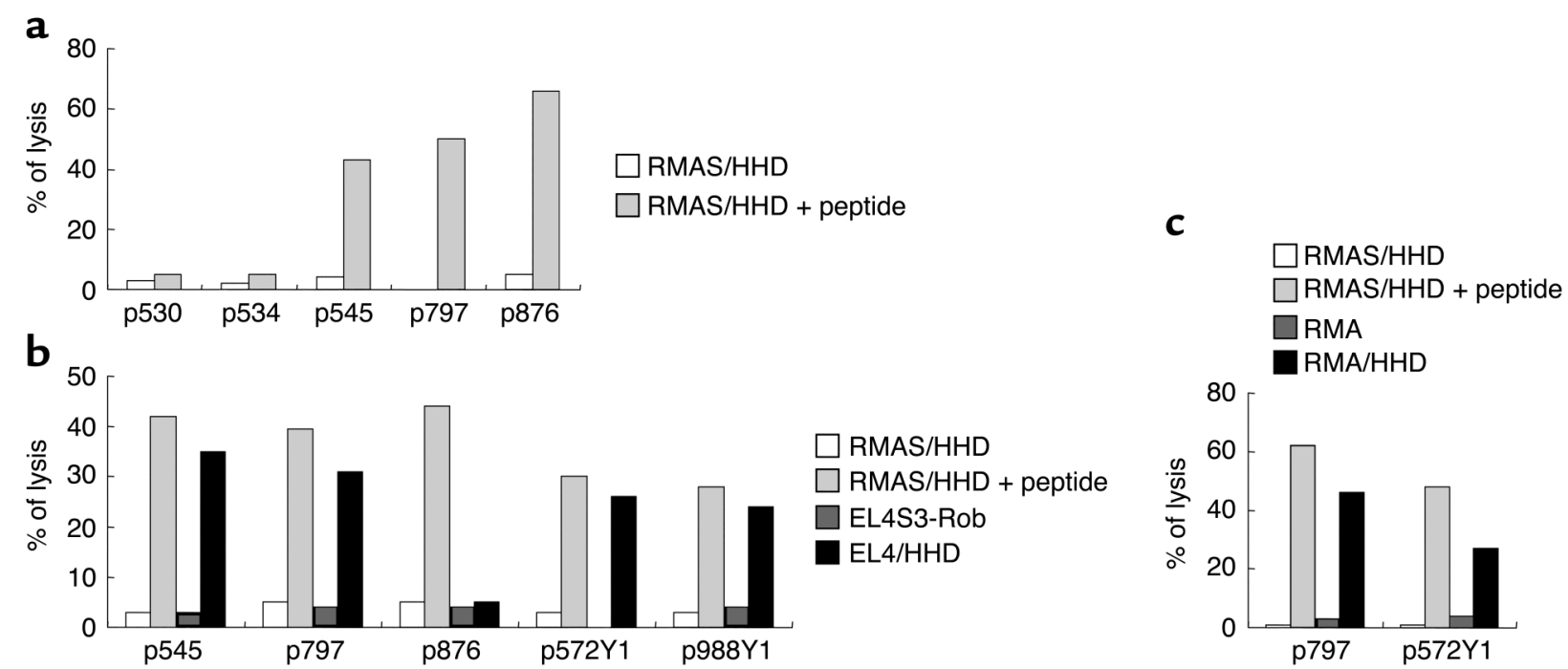

Figure 1

Recognition of murine tumor cells by CTLs raised against mTERT peptides with high and low affinity for HLA-A*0201. (a) HHD mice were vaccinated with mTERT high-affinity peptides, and their spleen cells were restimulated in vitro 11 days later with the cognate peptide. CTLs were tested against RMAS/HHD cells loaded with an irrelevant (white bars) or the cognate peptide (light gray bars) at a 40:1 lymphocyte/target ratio. Results from one representative mouse per group are shown. (b) $C T L_{545}, C T L_{797}, C T L_{876} C T L_{572}$, and $C T L_{988}$ lines were tested against RMAS/HHD loaded with an irrelevant or the cognate peptide, EL4S3-Rob, and EL4/HHD as indicated at a 20:1 lymphocyte/target ratio. (c) $C T L_{797}$ and $C T L_{572}$ lines were tested against RMAS/HHD loaded with an irrelevant or the cognate peptide, RMA, and RMA/HHD cells at a 20:1 lymphocyte/target ratio. 
a
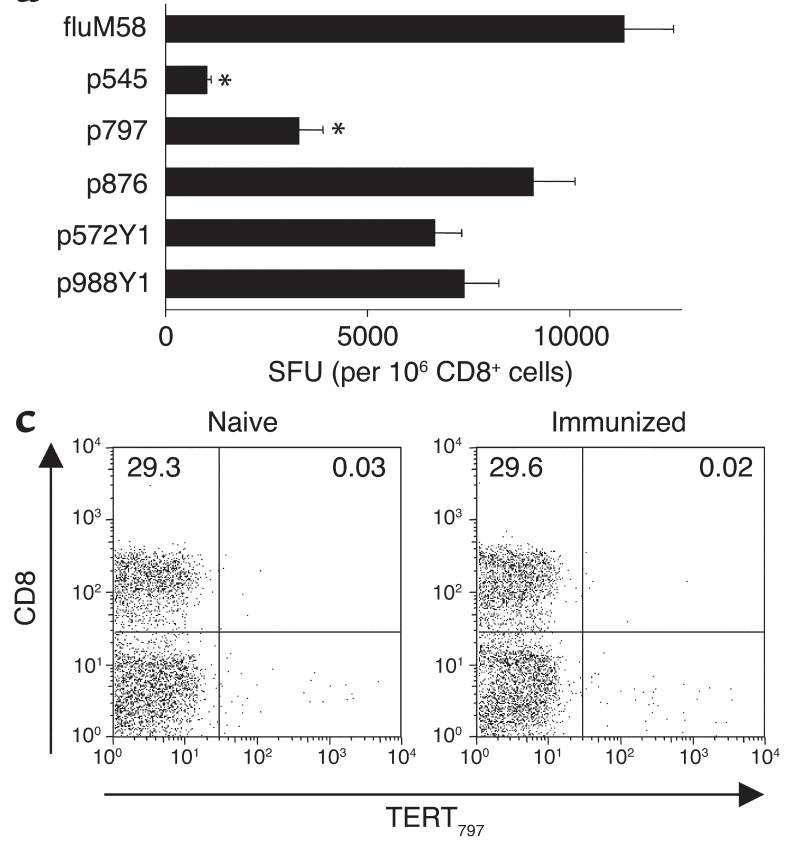

b

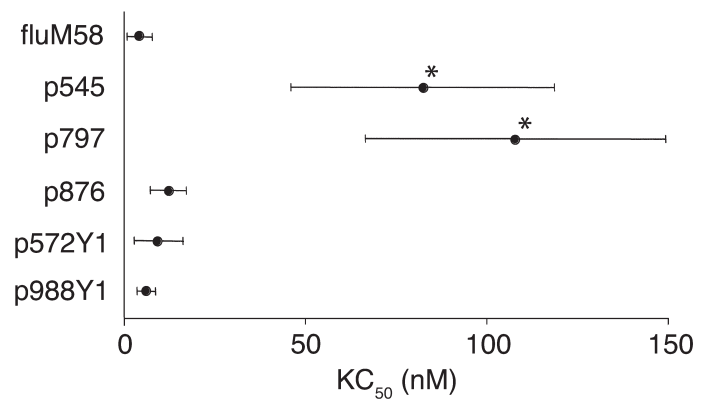

d

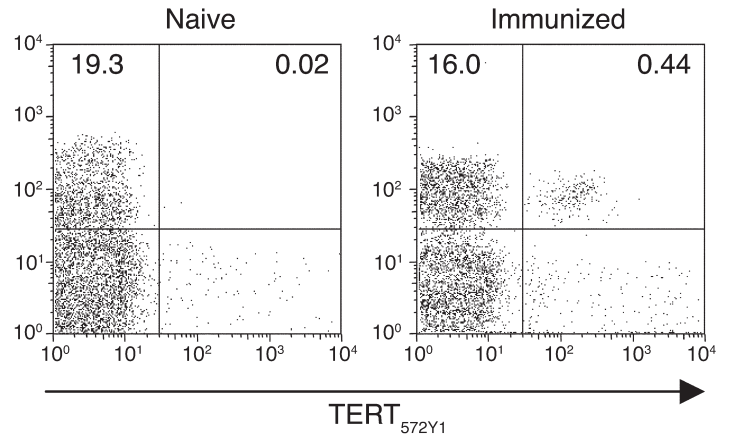

Figure 2

Quality of the mTERT epitope-specific CTL repertoire. From 5 to 10 mice were vaccinated with the high-affinity p545 and p797, the heteroclitical p572Y and p988Y, or the nonself controls p876 and fluM58 peptides. Results represent the mean per group and are expressed as mean \pm SEM. (a) CTL frequency was evaluated ex vivo 11 days after vaccination by a standard 24-hour IFN- $\gamma$ ELISPOT assay with cognate native peptides. Spot-forming units (SFUs) for $10^{6} \mathrm{CD} 8^{+}$splenocytes are represented. (b) Avidity of CTLs after 1 week of in vitro restimulation was defined by measuring the normalized concentration of native peptide that gives $50 \%$ of maximal lysis $\left(K_{50}\right)$. The normalized concentration was the ratio of peptide concentration to the RA. CTL frequency and $\mathrm{KC}_{50}$ were compared using the nonparametric Mann-Whitney $U$ test; asterisks in $\mathbf{a}$ and $\mathbf{b}$ indicate values that were considered significant $(P<0.05)$. (c) Peripheral blood mononuclear cells from naive or immunized mice 11 days earlier were stained with specific PE-coupled HLA-A*0201/p797 or (d) HLA-A*0201/p572Y tetramer together with anti-CD8-APC, anti-TCR $\beta-C y C h r o m e$, and anti-CD44-FITC. Representatives staining gated in TCR $\beta^{+}$CD $44^{+}$are shown.

peripheral tissues. To discriminate between these two phenomena, we used tetramers to detect antigen-specific peripheral blood $\mathrm{T}$ lymphocytes. Tetramers specific for $\mathrm{p} 797$ and $\mathrm{p} 572 \mathrm{Y}$ were first validated on specific CTL lines (data not shown). Significant responses were defined as those with a frequency of tetramer-positive cells above that found in naive mice plus three times the SD. With this criterion, none of the 9 mice immunized with the 7797 peptide presented a significant response (Figure 2c), whereas 8 out of 11 mice immunized with the $\mathrm{p} 572 \mathrm{Y}$ peptide presented a significant response (Figure $2 \mathrm{~d}$ ). The low avidity of the p797 repertoire combined with the absence of recognition of human tetramer by mouse CD8 may account for the lack of tetramer staining. In the case of the p572 epitope, we found similar amounts of CD8-specific cells by tetramer staining $\left(0.52 \% \pm 0.67 \%\right.$ of $\mathrm{CD}^{+}$cells $)$and by IFN- $\gamma$ ELISPOT assay $\left(0.65 \% \pm 0.06 \%\right.$ of $\mathrm{CD}^{+}$cells), suggesting that p572-specific cells are not anergic. As expected, nearly all tetramer-positive cells displayed an activated phenotype, as shown by the upregulation of the CD44 activation marker at their surface (data not shown).

Collectively, these results indicate that the CTL repertoire directed against low-affinity epitopes is poorly affected by central deletion.
In vivo tumor immunity induced by bigh-and low-affinity mTERT epitopes. To compare the vaccine efficacy of high- and low-affinity mTERT epitopes, we immunized HHD mice with p545, p797, p572Y, and p988Y once and then again after 2 weeks. HHD mice immunized with the irrelevant gp $100_{154}$ peptide were used as negative controls. At 7-10 days after the second immunization, we challenged mice with $2 \times 10^{4}$ EL4/HHD cells and monitored tumor growth and survival. The results (Figure 3a) show that at day 40 after challenge there was no difference in tumor size between mice vaccinated with high-affinity mTERT epitopes and the control gp $100_{154}$ peptide, suggesting that vaccination with the high-affinity p545 and p797 TERT epitopes does not induce a protective tumor immunity. In contrast, tumor size in tumor-bearing p572Y- and p988Y-vaccinated mice was significantly lower than in control mice $(P<0.0001)$. Furthermore, $33 \%$ and $25 \%$ of mice vaccinated with the $\mathrm{p} 572 \mathrm{Y}$ and $\mathrm{p} 988 \mathrm{Y}$ peptides, respective$l y$, were tumor free at this time point. Since slow tumor growth or metastases appearance cannot be monitored by tumor size only, survival was checked during the course of 3 months (Figure $3 b$ ). In fact, whereas all high-affinity mTERT epitope- or control-vaccinated mice died before day $55,25 \%$ of mice vaccinated with 

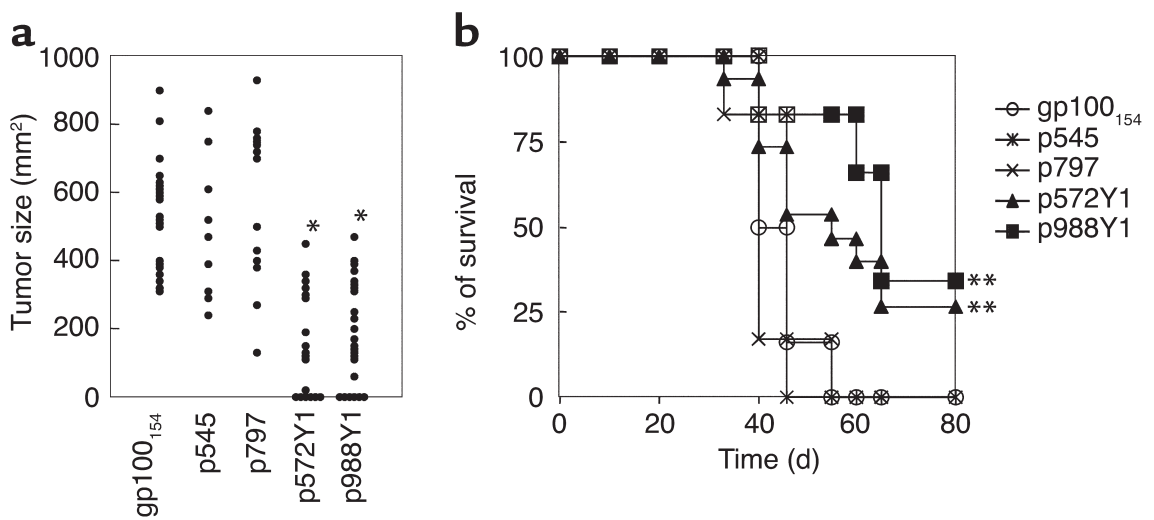

Figure 3

In vivo antitumor immunity induced in HHD mice vaccinated against mTERT high- and low-affinity epitopes. HHD mice were vaccinated with high-affinity or heteroclitical P1Y peptides once and then again 2 weeks later. Mice were challenged 7-10 days after the second vaccination with $2 \times 10^{4} \mathrm{EL} 4 / \mathrm{HHD}$ cells. The antitumor immunity was evaluated by measuring the tumor size $35-40$ days after challenge (a) and survival (b). Cumulative results from three independent experiments are shown. Tumor sizes in HHD mice vaccinated with $\mathrm{p} 988 \mathrm{Y}$ and $\mathrm{p} 572 \mathrm{Y}$ were statistically different from the tumor sizes in control HHD mice $(P<0.0001)$, as assessed by nonparametric Mann-Whitney $U$ test analysis $\left(^{*}\right)$. Survival statistically different from that of control gP100 154 -vaccinated HHD mice is indicated; ${ }^{*} P<0.03$ by Tarone and Ware test.

p572Y and p988Y were still alive at day $80(P<0.02)$. These results demonstrate that heteroclitical low-affinity TERT epitopes induce an antitumor protective immunity while high-affinity epitopes do not.

Vaccination with the heteroclitical low-affinity $p 572 Y$ epitope does not induce autoimmune phenomena. In mice, TERT is found in germ cells, hematopoietic progenitors, thymocytes, germinal-center B cells, activated lymphocytes, as well as in some vital solid organs including the liver, kidneys, and brain (25-27). To evaluate the potential autoreactivity against low-affinity TERT peptides as induced by heteroclitical peptides, we first tested whether CTLs generated by the heteroclitical p572Y peptide recognize such mTERT-expressing normal cells as activated B cells. Results presented in Figure 4a show that the $\mathrm{CTL}_{572}$ line does not kill activated $B$ cells from HHD mice, confirming our previous data suggesting that human CTLs against p572 do not kill activated HLA-A*0201+ B cells (unpublished data). The absence of reactivity against normal cells, such as activated B cells, could be attributed to low expression of TERT in normal cells compared with tumor cells. However, mTERT-specific mAb's are not available to test this hypothesis directly. Instead, we performed a real-time PCR TRAP-modified assay to quantify telomerase activity, since the TERT subunit is the limiting factor for this activity (35). We found that RMA/HHD and EL4/HHD cells exhibit 10- to 20-fold higher levels of telomerase activity than LPS-activated B cells or ConA-activated T cells, and 100fold higher levels than bone marrow cells (Figure $4 \mathrm{~b}$ ), in correspondence with differential sensibility to CTL lysis.
Since our results obtained in vitro argued against potential self-reactivity of p572-specific CTLs, we further checked in vivo the presence of autoimmune reactions. HHD mice vaccinated with $\mathrm{p} 572 \mathrm{Y}$ and the control gp $100_{154}$ peptides were examined histologically (Figure 5a) and immunohistologically (data not shown) at different time points (from 6 weeks to 1 year) after vaccination. At 6 months after vaccination, splenic B-cell follicles retained their normal architecture and cellular compartmentalization. The lymph nodes, Peyer's patches, and thymus were normal. The liver was also normal, without any lymphoid infiltration. Similar results were obtained 1 year after vaccination. Moreover, phenotypic analysis of spleen cells did not show any marked diminution of $\operatorname{IgM}^{\text {high }} \operatorname{IgD}^{\text {low }}$-acti-
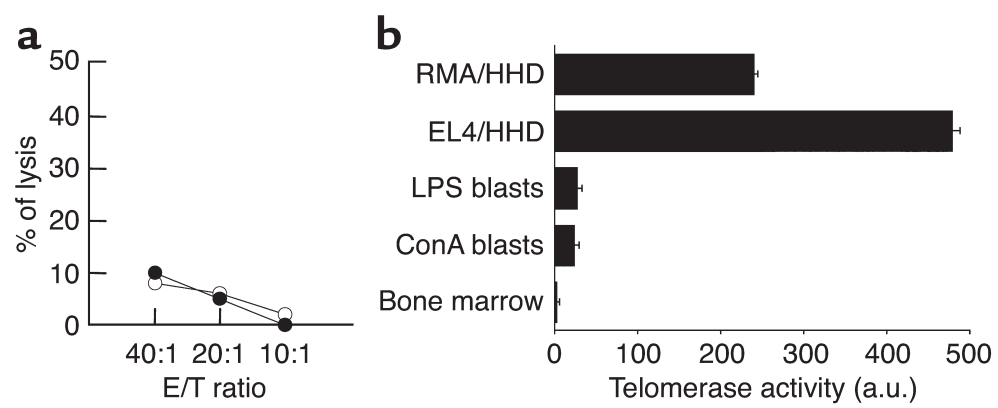

Figure 4

Susceptibility to cell lysis by $C T L_{572}$ correlates with telomerase activity in vitro. (a) $\mathrm{CTL}_{572}$ line was tested against LPS-activated B cells from HHD (filled circles) and C57BL6 (open circles) mice. E/T ratio, effector/target ratio. (b) Quantification of telomerase activity in normal cells and in tumor cell lines. Telomerase activity was measured by real-time quantitative TRAP assay. Results are calculated from the HL60 cell standard curve and expressed in arbitrary units (a.u.) of telomerase activity defined as the activity of $10^{3} \mathrm{HL} 60$ cells. Experiments were done in duplicate, and results are expressed as mean \pm SD. SDs are always less than $1 \%$ of the mean. Mononuclear cells were obtained from HHD bone marrow and LPS or ConA blasts from HHD-activated splenocytes. 

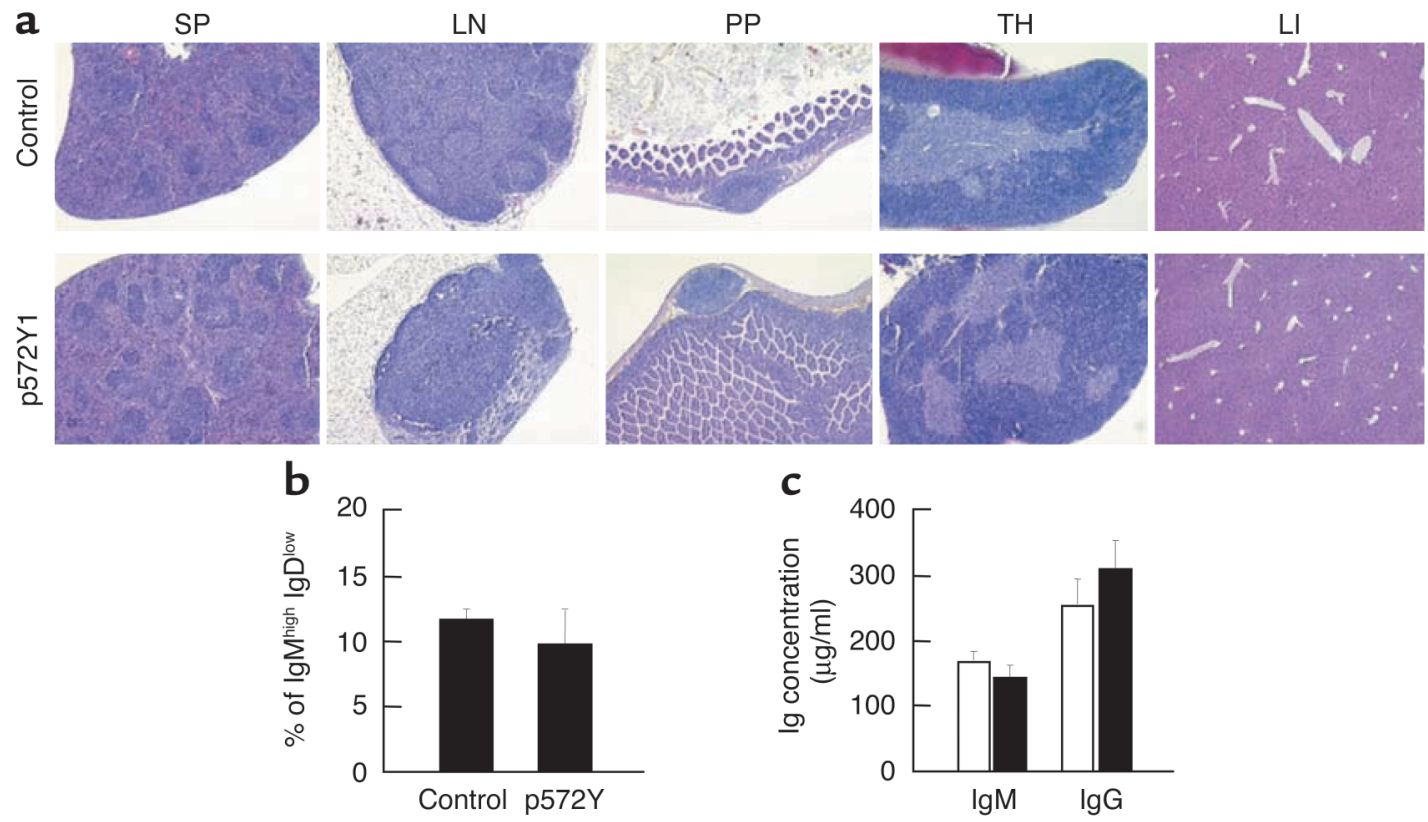

\section{Figure 5}

Absence of autoimmune reactivity against TERT-expressing normal cells and tissues in HHD mice vaccinated with p572Y. (a) Histological analysis of TERT-expressing normal tissues in HHD mice vaccinated with the control gp $100_{154}$ or the p572Y peptide. TERT-expressing spleen (SP), lymph nodes (LN), gut (PP), thymus (TH), and liver (LI) were histologically examined 6 months later. H\&E staining. (b) Spleen cells from naive HHD mice vaccinated with gp100 154 (control) or with the p572Y peptide 6 months earlier were stained with anti-B220-APC, antiIgD-FITC, anti-IgM-PE. The double anti-IgD and anti-IgM analysis was evaluated for the B220+ cell population. (c) IgM and IgG were measured by ELISA in the serum of control HHD mice (white bars) or HHD mice vaccinated with the p572Y peptide 6 months earlier (black bars).

vated B cells (Figure $5 b$ ), and serum IgM and IgG levels were normal (Figure 5c). Finally, cell counts of peripheral blood granulocytes, lymphocytes, red cells, and platelets were normal up to 4 months after vaccination with the p572Y peptide (data not shown).

These results, taken together, demonstrate that vaccination with the heteroclitical p572Y cryptic mTERT epitope does not induce autoreactivity targeting normal tissues expressing low amounts of mTERT.

\section{Discussion}

We have explored here the antitumor vaccination efficiency of TAA epitopes of high and low affinity for the MHC class I molecule, as a rationale to target a nontolerized $\mathrm{T}$ cell repertoire against naturally expressed tumor antigens. As a model, we analyzed the HLA$A^{*} 0201$ response to the widely expressed TERT tumor antigen in a fully homologous mouse system. We found that the CTL repertoire against the high-affinity mTERT epitopes is either completely (p530 and p534) or partially (p545 and p797) tolerized, whereas CTLs specific for low-affinity epitopes (p572 and p988) are weakly if at all affected by tolerogenic mechanisms. The large size and high avidity of their repertoire correlate with their antitumor efficacy. These results extend to a tumor response setting, previous reports showing that self-tolerance operates mainly on the $T$ cell repertoire directed against dominant self-epitopes and not to that against cryptic determinants $(18,19)$. Even if it is hazardous to predict crypticity or domi- nance for self-antigen and TAAs, we found that HLA affinity/stability is a highly discriminating parameter that is instrumental in identifying efficient tumor rejection epitopes within the TERT sequence.

Previous reports indicated that high-affinity tumor epitopes derived from autologous tumor antigens do not necessarily induce efficient tumor rejection using peptide-based vaccination protocols $(36,37)$. In the case of TAAs normally expressed in healthy tissues, selftolerance shapes the TAA repertoire and purges the high-avidity CTLs. The remaining $\mathrm{T}$ cell specificities of weak avidity are insufficient to elicit antitumor immunity, as shown for CTL responses to p53 and tyrosinase induced after peptide vaccination $(3,4)$. These results led us to revisit the antitumor vaccination potential of the nontolerized repertoire directed against low-affinity epitopes of TAAs. Although these epitopes are nonimmunogenic and then disregarded for antitumor approaches, we showed previously that HLA-A*0201restricted peptides become highly immunogenic when modified with a tyrosine in first position (P1Y) (23). Many peptide variants have been used in the past to improve MHC class I affinity, but these peptides were mostly derived from intermediate or good HLA binders (gP100 209 , MART- $1_{26}$ ) modified at the anchor positions $\mathrm{P} 2$ and/or P9/10 $(38,39)$. These peptide variants can trigger strong CTL responses in vitro (38) and in mice (40), and they induced efficient antitumor immunity in cancer patients when associated with IL-2 (41). Here we used P1Y variants to explore the CTL repertoire 
directed against low-affinity epitopes. A recent report has demonstrated the feasibility of this approach using xenogeneic vaccination with human gp100 DNA to induce tumor growth protection in mice (42). Indeed, these authors found that the high-affinity and dominant epitope of human gp100, corresponds to a lowaffinity and cryptic epitope of mouse gp100, and recruits a nontolerized CTL repertoire.

The question raised by our observations is whether one should avoid peptide vaccination with high-affinity tumor epitopes in tumor immunotherapy. Highavidity CTLs specific for high-affinity dominant tumor epitopes have been recruited in some circumstances. For instance, high-avidity CTLs against a high-affinity tyrosinase epitope were obtained from splenocytes of vaccinated mice once they were restimulated with low doses of peptide in vitro (4). Here, the CTL797.3 clone established in similar conditions, despite partial tolerization against the high-affinity p797 epitope, exhibits a higher avidity $(10 \mathrm{nM})$ than the bulk CTL population (data not shown). Thus, vaccination protocols that can recruit these rare high-avidity CTLs are primordial for the use of high-affinity epitopes in tumor immunotherapy. With this respect, dendritic cells loaded with peptides or whole antigens are certainly essential to recruit the rare high-avidity naive $\mathrm{T}$ cells. Indeed, it was recently reported that mice immunized with the $\mathrm{Db}$ restricted WT1 or Kb-restricted TRP2 high-affinity epitopes loaded on dendritic cells are protected against challenge with tumor cells expressing the corresponding antigen, whereas immunization with high-affinity peptides in IFA does not confer protection $(36,37)$. Another important report indicates that antitumor immunity against TERT can be implemented in vivo using TERT RNA-transfected dendritic cells (32). A strong effect on tumor size was observed, but the percentage of tumor-free animals amounted to $1 / 15$ at day 28. In our experiments, low-affinity epitope vaccination also provided decreased tumor size, and 4/15 animals were tumor-free for each epitope at 5-6 weeks. Since many approaches are superior to IFA adjuvant to override tolerance to self, we propose that combining them with heteroclitical peptides will induce higher levels of tumor protection.

Finally, the hallmark of efficient antitumor immune reactions is often autoimmunity. Limited vitiligo reactions have been reported after melanoma-associated antigen vaccination $(4,43)$, even in humans (44). In contrast, adoptive transfer of high-avidity p53-specific CTLs into p53 WT mice prevents p53-overexpressingtumor outgrowth in the absence of any demonstrable damage to normal tissues (45). A notable feature of our study is that mice vaccinated with the heteroclitical cryptic $\mathrm{p} 572 \mathrm{Y}$ peptide showed no sign of autoimmune reactions. Although vaccination with $\mathrm{p} 572 \mathrm{Y}$ peptide led to the generation of high-avidity specific CTLs in vivo that are protective against tumor growth, TERTexpressing B cells and other normal tissues were not markedly affected in vivo. These results are consistent with those observed after vaccination with TERT RNAtransfected dendritic cells (32). A possible explanation is that TERT expression is insufficient in normal cells to allow presentation of peptide with low affinity (e.g., p572), while its expression is sufficient in tumor cells. From telomerase activity measurements, our murine tumor cells probably express higher amounts of TERT than normal hematopoietic cells, indicating that the susceptibility to CTL lysis is correlated with TERT expression. Moreover, after the first wave of CTL response driven by low-affinity epitopes, partial tumor destruction may lead to epitope spreading, which targets tumor-specific antigens $(46,47)$.

Our results demonstrate that low-affinity epitopes from universal self tumor antigens, such as TERT, are more efficient at inducing tumor immunity than highaffinity epitopes and do not induce autoimmune reactions. It has recently been shown that the tumor itself may have an impact since in chronic myelogenous leukemia patients, the avidity of the CTLs directed against the high-affinity PR1 epitope is lower than in healthy individuals (48). Low-affinity epitopes of universal tumor antigens are therefore promising targets for tumor immunotherapy applications in humans.

\section{Acknowledgments}

This work was supported by grants from l'Institut National de la Santé et de la Recherche Médicale (Progres 4P006E), l'Institut Gustave Roussy (74888M), and l'Association pour la Recherche sur le Cancer (5909). The authors thank D. Chaluau and E. Connault for technical assistance in in vivo experiments and histological studies.

1. Van Pel, A., et al. 1995. Genes coding for tumor antigens recognized by cytolytic T lymphocytes. Immunol. Rev. 145:229-250.

2. Robbins, P.F., and Kawakami, Y. 1996. Human tumor antigens recognized by T cells. Curr. Opin. Immunol. 8:628-636.

3. Theobald, M., et al. 1997. Tolerance to p53 by A2.1-restricted cytotoxic T lymphocytes. J. Exp. Med. 185:833-841.

4. Colella, T.A., et al. 2000. Self-tolerance to the murine homologue of a tyrosinase-derived melanoma antigen: implications for tumor immunotherapy. J. Exp. Med. 191:1221-1232.

5. Alexander-Miller, M.A., Leggatt, G.R., and Berzofsky, J.A. 1996. Selective expansion of high- or low-avidity cytotoxic T lymphocytes and efficacy for adoptive immunotherapy. Proc. Natl. Acad. Sci. U. S. A. 93:4102-4107.

6. Perez-Diez, A., Spiess, P.J., Restifo, N.P., Matzinger, P., and Marincola, F.M. 2002. Intensity of the vaccine-elicited immune response determines tumor clearance. J. Immunol. 168:338-347.

7. Zeh, H.J., III, Perry-Lalley, D., Dudley, M.E., Rosenberg, S.A., and Yang, J.C. 1999. High avidity CTLs for two self-antigens demonstrate superior in vitro and in vivo antitumor efficacy. J. Immunol. 162:989-994.

8. van Elsas, A., Hurwitz, A.A., and Allison, J.P. 1999. Combination immunotherapy of B16 melanoma using anti-cytotoxic T lymphocyteassociated antigen 4 (CTLA-4) and granulocyte/macrophage colonystimulating factor (GM-CSF)-producing vaccines induces rejection of subcutaneous and metastatic tumors accompanied by autoimmune depigmentation. J. Exp. Med. 190:355-366.

9. Liu, X., et al. 2001. B7H costimulates clonal expansion of, and cognate destruction of tumor cells by, CD8(+) T lymphocytes in vivo. J. Exp. Med. 194:1339-1348.

10. Sutmuller, R.P., et al. 2001. Synergism of cytotoxic T lymphocyte-associated antigen 4 blockade and depletion of CD25(+) regulatory $\mathrm{T}$ cells in antitumor therapy reveals alternative pathways for suppression of autoreactive cytotoxic T lymphocyte responses. J. Exp. Med. 194:823-832.

11. Smyth, M.J., Godfrey, D.I., and Trapani, J.A. 2001. A fresh look at tumor immunosurveillance and immunotherapy. Nat. Immunol. 2:293-299.

12. Zitvogel, L., et al. 1998. Eradication of established murine tumors using a novel cell-free vaccine: dendritic cell-derived exosomes. Nat. Med. 4:594-600. 
13. Blachere, N.E., et al. 1997. Heat shock protein-peptide complexes, reconstituted in vitro, elicit peptide-specific cytotoxic T lymphocyte response and tumor immunity. J. Exp. Med. 186:1315-1322.

14. Thomas, C.E., Ehrhardt, A., and Kay, M.A. 2003. Progress and problems with the use of viral vectors for gene therapy. Nat. Rev. Genet. 4:346-358.

15. Mocellin, S., Rossi, C.R., Lise, M., and Marincola, F.M. 2002. Adjuvant immunotherapy for solid tumors: from promise to clinical application. Cancer Immunol. Immunother. 51:583-595.

16. Rock, K.L., and Goldberg, A.L. 1999. Degradation of cell proteins and the generation of MHC class I-presented peptides. Annu. Rev. Immunol. 17:739-779.

17. Moudgil, K.D., and Sercarz, E.E. 2000. The self-directed T cell repertoire: its creation and activation. Rev. Immunogenet. 2:26-37.

18. Cibotti, R., et al. 1992. Tolerance to a self-protein involves its immunodominant but does not involve its subdominant determinants. Proc. Natl. Acad. Sci. U. S. A. 89:416-420.

19. Nanda, N.K., and Sercarz, E.E. 1995. Induction of anti-self-immunity to cure cancer. Cell. 82:13-17.

20. Pascolo, S., et al. 1997. HLA-A2.1-restricted education and cytolytic activity of CD8(+) T lymphocytes from $\beta 2$ microglobulin ( $\beta 2 \mathrm{~m}$ ) HLA-A2.1 monochain transgenic $\mathrm{H}-2 \mathrm{Db} \beta 2 \mathrm{~m}$ double knockout mice. J. Exp. Med. 185:2043-2051.

21. Miyahira, Y., et al. 1995. Quantification of antigen specific CD8+ T cells using an ELISPOT assay. J. Immunol. Methods. 181:45-54.

22. Tourdot, S., et al. 1997. Chimeric peptides: a new approach to enhancing the immunogenicity of peptides with low MHC class I affinity: application in antiviral vaccination. J. Immunol. 159:2391-2398.

23. Tourdot, S., et al. 2000. A general strategy to enhance immunogenicity of low-affinity HLA-A2. 1-associated peptides: implication in the identification of cryptic tumor epitopes. Eur. J. Immunol. 30:3411-3421.

24. Scardino, A., et al. 2002. HER-2/neu and hTERT cryptic epitopes as novel targets for broad spectrum tumor immunotherapy. J. Immunol. 168:5900-5906.

25. Prowse, K.R., and Greider, C.W. 1995. Developmental and tissue-specific regulation of mouse telomerase and telomere length. Proc. Natl. Acad. Sci. U. S. A. 92:4818-4822.

26. Liu, K., et al. 1999. Constitutive and regulated expression of telomerase reverse transcriptase (hTERT) in human lymphocytes. Proc. Natl. Acad. Sci. U. S. A. 96:5147-5152.

27. Ramakrishnan, S., Eppenberger, U., Mueller, H., Shinkai, Y., and Narayanan, R. 1998. Expression profile of the putative catalytic subunit of the telomerase gene. Cancer Res. 58:622-625.

28. Vonderheide, R.H., Hahn, W.C., Schultze, J.L., and Nadler, L.M. 1999. The telomerase catalytic subunit is a widely expressed tumor-associated antigen recognized by cytotoxic T lymphocytes. Immunity. 10:673-679.

29. Vonderheide, R.H., et al. 2001. Characterization of HLA-A3-restricted cytotoxic $\mathrm{T}$ lymphocytes reactive against the widely expressed tumor antigen telomerase. Clin. Cancer Res. 7:3343-3348.

30. Arai, J., et al. 2001. Identification of human telomerase reverse transcriptase-derived peptides that induce HLA-A24-restricted antileukemia cytotoxic T lymphocytes. Blood. 97:2903-2907.

31. Schroers, R., Huang, X.F., Hammer, J., Zhang, J., and Chen, S.Y. 2002. Identification of HLA DR7-restricted epitopes from human telomerase reverse transcriptase recognized by CD4+ T-helper cells. Cancer Res. 62:2600-2605

32. Nair, S.K., et al. 2000. Induction of cytotoxic T cell responses and tumor immunity against unrelated tumors using telomerase reverse transcriptase RNA transfected dendritic cells. Nat. Med. 6:1011-1017.

33. Delhommeau, F., et al. 2002. Telomere dysfunction and telomerase reactivation in human leukemia cell lines after telomerase inhibition by the expression of a dominant-negative hTERT mutant. Oncogene. 21:8262-8271.

34. Parker, K.C., Bednarek, M.A., and Coligan, J.E. 1994. Scheme for ranking potential HLA-A2 binding peptides based on independent binding of individual peptide side-chains. J. Immunol. 152:163-175.

35. Counter, C.M., et al. 1998. Telomerase activity is restored in human cells by ectopic expression of hTERT (hEST2), the catalytic subunit of telomerase. Oncogene. 16:1217-1222.

36. Gaiger, A., Reese, V., Disis, M.L., and Cheever, M.A. 2000. Immunity to WT1 in the animal model and in patients with acute myeloid leukemia. Blood. 96:1480-1489.

37. Schreurs, M.W., et al. 2000. Dendritic cells break tolerance and induce protective immunity against a melanocyte differentiation antigen in an autologous melanoma model. Cancer Res. 60:6995-7001.

38. Valmori, D., et al. 1998. Enhanced generation of specific tumor-reactive CTL in vitro by selected Melan-A/MART-1 immunodominant peptide analogues. J. Immunol. 160:1750-1758.

39. Parkhurst, M.R., et al. 1996. Improved induction of melanoma-reactive CTL with peptides from the melanoma antigen gp100 modified at HLAA*0201-binding residues. J. Immunol. 157:2539-2548.

40. Men, Y., et al. 1999. Assessment of immunogenicity of human Melan-A peptide analogues in HLA-A*0201/Kb transgenic mice. J. Immunol. 162:3566-3573.

41. Rosenberg, S.A., et al. 1998. Immunologic and therapeutic evaluation of a synthetic peptide vaccine for the treatment of patients with metastatc melanoma. Nat. Med. 4:321-327.

42. Overwijk, W.W., et al. 1998. gp100/pmel 17 is a murine tumor rejection antigen: induction of "self"-reactive, tumoricidal T cells using highaffinity, altered peptide ligand. J. Exp. Med. 188:277-286.

43. Overwijk, W.W., et al. 1999. Vaccination with a recombinant vaccinia virus encoding a "self" antigen induces autoimmune vitiligo and tumor cell destruction in mice: requirement for CD4(+) T lymphocytes. Proc. Natl. Acad. Sci. U. S. A. 96:2982-2987.

44. Yee, C., et al. 2000. Melanocyte destruction after antigen-specific immunotherapy of melanoma: direct evidence of T cell-mediated vitiligo. J. Exp. Med. 192:1637-1644.

45. Vierboom, M.P., et al. 1997. Tumor eradication by wild-type p53-specific cytotoxic T lymphocytes. J. Exp. Med. 186:695-704.

46. el-Shami, K., et al. 1999. MHC class I-restricted epitope spreading in the context of tumor rejection following vaccination with a single immunodominant CTL epitope. Eur. J. Immunol. 29:3295-3301.

47. Brossart, P., et al. 2000. Induction of cytotoxic T-lymphocyte responses in vivo after vaccinations with peptide-pulsed dendritic cells. Blood. 96:3102-3108.

48. Molldrem, J.J., et al. 2003. Chronic myelogenous leukemia shapes host immunity by selective deletion of high-avidity leukemia-specific $\mathrm{T}$ cells. J. Clin. Invest. 111:639-647. doi:10.1172/JCI200316398. 